\title{
A Swarm Intelligence Inspired Algorithm for Contour Detection in Images
}

\author{
Ulrich Kirchmaier, Simon Hawe, Klaus Diepold \\ Lehrstuhl für Datenverarbeitung \\ Technische Universität München \\ Arcisstr. 21, 80290 Munich, Germany
}

\begin{abstract}
Swarm Intelligence uses a set of agents which are able to move and gather local information in a search space and utilize communication, limited memory, and intelligence for problem solving. In this work, we present an agent-based algorithm which is specifically tailored to detect contours in images. Following a novel movement and communication scheme, the agents are able to position themselves distributed over the entire image to cover all important image positions. To generate global contours, the agents examine the local windowed image information, and based on a set of fitness functions and via communicating with each other, they establish connections. Instead of a centralized paradigm, the global solution is discovered by some principal rules each agent is following. The algorithm is independent of object models or training steps. In our evaluation we focus on boundary detection as a major step towards image segmentation. We therefore evaluate our algorithm using the Berkeley Segmentation Dataset (BSDS) and compare its performance to existing methods via the BSDS benchmark and Pratt's Figure of Merit.
\end{abstract}

\section{Introduction}

Boundary Detection is commonly regarded as a major step towards Image Segmentation, which is a central problem in computer vision that has been studied for years. Image Segmentation again constitutes an important initial step for high-level tasks in computer vision, like object recognition, image analysis, and scene understanding. It pursues the goal of dividing an image into parts, regions, or objects, which are preferably self-consistent and of meaningful content. Application examples include separating objects considered as foreground from the background, or separating multiple objects from each other.

One of the main difficulties in the field of boundary detection and image segmentation arises from the fact, that not even human beings are able to uniquely and unambiguously decide and agree over a correct segmentation [1]. Different persons are likely to have different opinions on the correct solution to the segmentation problem of any image, especially involving natural images. This lack of an unique ground-truth complicates the comparison of results descending form different segmentation methods. Nevertheless, efforts were taken to establish a framework for objective evaluation of segmentation algorithms. A prominent example is the Berkeley Segmentation Dataset (BSDS) [1], which provides a huge collection of natural images along with human-marked proposals of ground-truth, as well as a precision-recall based measurement for comparison [2]. Figure 1 shows three example images along with three different human ground-truth suggestions.

The plenty of solutions to the segmentation problem proposed in the literature descend from versatile fields, such as clustering [4], graph theory [5, 6], region growing [7], optimization of an evaluation function like energy minimization [8], as well as image feature-based methods $[9,10]$. The majority of approaches found in the 

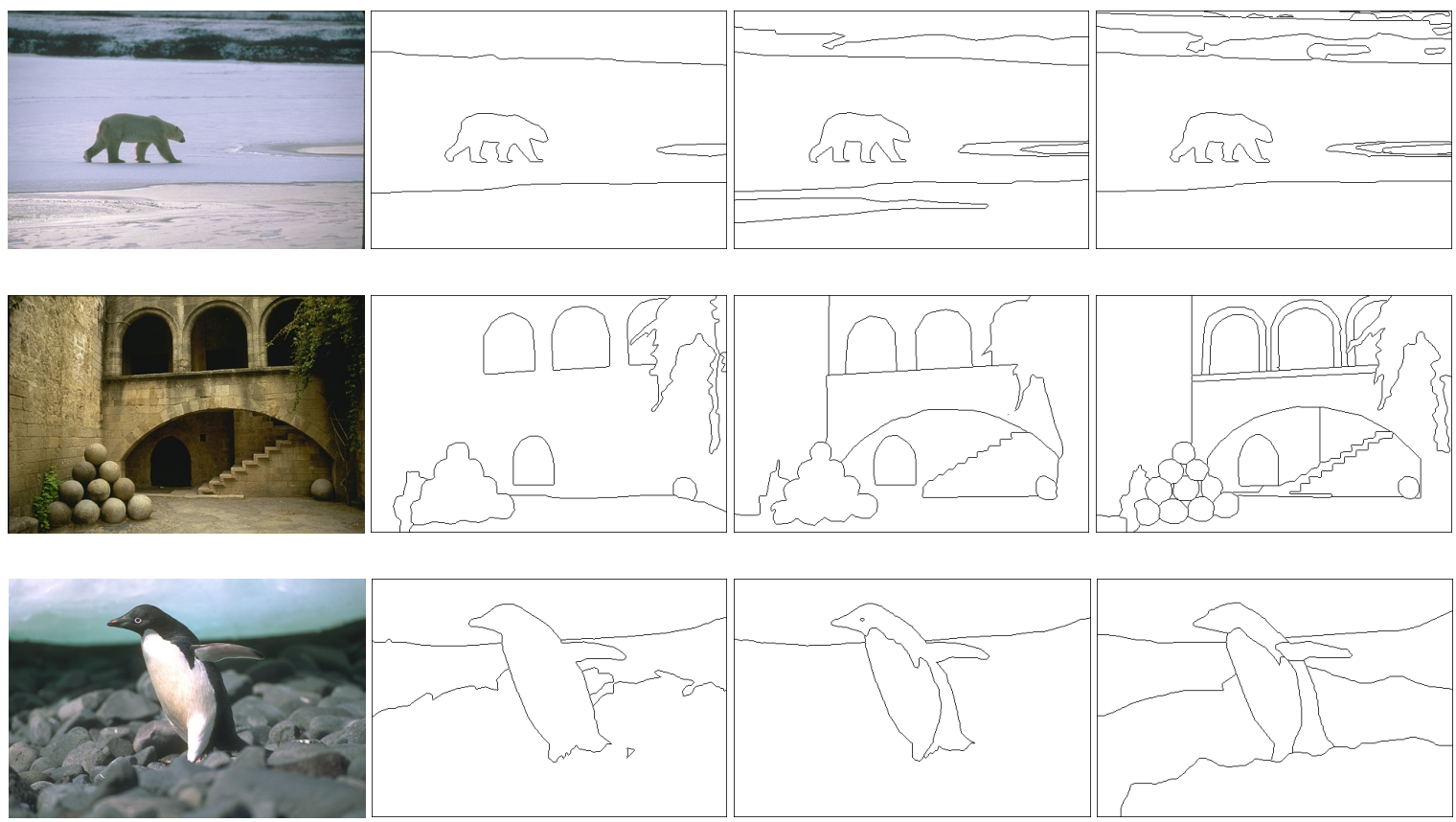

Figure 1: Three sample images from the Berkeley Image Segmentation Dataset (BSDS 500) [3], each along with three different human-marked ground-truth images.

literature can be categorized into one or a subset of these fields, with each adding improved mechanisms to solve specific tasks.

Boundary or contour detection is often performed as an initial step for image segmentation, as boundaries naturally correspond to borders between objects. It describes the process of identifying and locating sharp discontinuities in image regions, which closely relates it to the task of edge detection. Mere edge detection, however, registers on the one hand any type of abrupt changes in image brightness, and misses smooth or subtle transitions of image brightness on the other hand. Regarding the goal of detecting boundaries, this causes false positives like textured regions, or single highlighted spots and true negatives like region boundaries of low contrast.

One recipe to overcome these drawbacks is to combine edge detection techniques with diverse soft computing approaches like Genetic Algorithms, or Fuzzy Logic, etc. [11, 12]. Other methods combine oriented local image cues, like brightness, color, and texture descriptors with edge detection to get a global estimation. Such combination methods include multiscale cue combination [3], energy and probability weighting [13], or likelihood-methods [14].

In this paper, we propose an algorithm that uses the concept of Swarm Intelligence to detect contours in images. We believe that the idea of interacting distributed agents to migrate from local information to global boundary estimates is a promising alternative to existing methods.

While a variety of Swarm Intelligence inspired algorithms already exists (refer to Section 2), their swarms often seek to find one global optimal solution in a search space, as these algorithms descend from the field of optimization.

However, it is our goal to detect a non-predefined number of boundaries with arbitrary positions, sizes, and shapes in an image, which all have to be considered as equal or even independent solutions. Therefor, we established a novel algorithm based on distributed agents being explicitly tailored to the demands of contour detection. The main contribution of this paper are the development of (1) a novel movement and positioning rule, along with (2) local information gathering mechanisms, and (3) a set of fitness functions and communication schemes.

This paper is organized as follows: Section 2 gives an overview on approaches in the fields of image segmen- 
tation and contour detection, which utilize Swarm Intelligence. Section 3 investigates the principles of Swarm Intelligence and Section 4 provides details on our algorithm. Section 5 shows results of our algorithm tested on the task of boundary detection in natural images.

\section{Related Work}

In recent years, algorithms descending from the field of Evolutionary Computing, Genetic Algorithms, and also Swarm Intelligence are increasingly applied to the image segmentation problem. These optimization tools have mostly been used to improve the performance of important steps of segmentation algorithms. In [15, 16], evolutionary and genetic algorithms were used to enhance the clustering process in segmentation. In [17], the authors combined high-level features generated with a visual attention model with low-level features to guide region growing algorithm, where the optimal thresholds of the region growing process were detected using the Particle Swarm Optimization (PSO) algorithm.

In [18], the PSO was applied to find the optimal fuzzy entropy thresholds to segment images into foreground and background. A related approach was introduced in [19], where PSO was used to tune thresholds in 2Dhistograms, maximizing the entropy to segment infrared images.

SI was also utilized to improve Segmentation based on clustering approaches, which can get stuck in local optima, depending on their initialization. In [20], the authors developed a hybrid combination of the Ant Colony Optimization algorithm (ACO) and PSO to make K-Means clustering and Single Competitive Learning more independent from their initial cluster centers, and learning rate, respectively.

Besides deploying SI techniques to optimize specific steps in existing segmentation approaches, SI algorithms were also applied to the tasks of edge detection and contour detection, akin to our proposed algorithm.

The authors of [21] proposed ant-based correlation for edge detection. Their method is capable of performing feature extraction for edge detection and segmentation, generating less distortion in the presence of noise, as compared to classical edge detectors, like Sobel, Prewitt, and Canny.

In [22], a variant of the PSO algorithm is utilized for finding a proper edge detection filter size in noisy images. While combining edge filters of different sizes can improve the edge detection in noisy images, it is generally computationally expensive when applied to the entire image. Thus, the heuristic capabilities of PSO were used to adaptively decide, where on the image to use filters of various sizes. The evaluation of edge intensity and the proposed movement principle in this approach has analogies to our algorithm, as they divide the edge regions into sets and use averaged intensities. However, the major difference is the usage of discrete PSO instead of our communication scheme where the agents share their local information.

\section{Principles of Swarm Intelligence}

Swarm intelligence constitutes a fast-growing field with researchers from various disciplines from social and natural sciences to computational engineering engaged. In nature, phenomena of SI can be found in multiple examples, including fish schooling, ant colonies, bird flocking, animal herding, and bacterial growth, as well as human crowd behavior.

$\mathrm{SI}$ is based on self-organized individuals, generally called agents, whose actions and interaction add up to intelligent global behavior. This occurrence is commonly referred to as emergence. Thereby, a key point of SI is the absence of a centralized control instance. Instead, each agent acts according to some basic principles, which are

- autonomy, i.e. no centralized control,

- local view, i.e. incomplete knowledge of the environment and also no or little concern about the global behavior of the system,

- movement, i.e. an agent's possibility to discover its environment,

- communication, i.e. interaction between agents,

- memory, i.e. knowledge of prior conditions,

- fitness function, i.e. a measure to evaluate an agents current position or solution. 
Since the first appearance of SI in a technical context [23], researchers utilized its principles on various applications, for optimization, and data analysis problems [24]. In recent years, SI-based methods proofed to be capable of managing complex systems and tasks, from route-planning and control of unmanned vehicles to mobile telecommunication networks.

While SI-based algorithms mostly descend from the field of optimization, they were also successfully applied to computer vision and image processing tasks, ranging from edge detection [25], over feature extraction and object recognition [26, 27], to three-dimensional tracking in stereo-video [28]. All these algorithms offer robustness alongside low computational costs.

Two prominent and widely-used examples for realizations of the SI-principles are the Particle Swarm Optimization (PSO) [29], and the Ant Colony Optimization ACO [30]. These two instances show the manifold possibilities of interpretation of the SI-concept. While they follow the same principles, their realizations illustrate significant differences. To give an example, the communication of the ACO algorithm is indirect and locationbased, i.e. the ants leave a pheromone-trail at each location, which describes the position with respect to the task. On the opposite, the particles of PSO use a one-to-all broadcast-communication, which causes the other particles of the swarm to move closer to the currently best solution.

Algorithms of the SI-field have been already applied to image segmentation in various ways, e.g. for finding adaptive thresholds of regions using PSO [31], or on color segmentation via an ACO-based algorithm using a specialized swarm of scouts and workers [32]. In [33], an agent-based diffusion-breeding concept was introduced to image segmentation, in which the agents move over an image and connect homogeneous regions via an interplay of creating offspring and deactivating agents.

In this work, we present a novel algorithm based on the principles of Swarm Intelligence, that is particularly tailored to the task of contour or boundary detection in images. Boundaries in images can be arbitrarily distributed over the entire image, hence an algorithm should be able to detect and localize them independent of their position, size, shape, orientation, and appearance. The distributed nature of the swarms established in this algorithm suits to this circumstance. The details of our algorithm are explained in the following Section.

\section{SI Contour Detection Algorithm}

Following the principles of Swarm Intelligence mentioned in the last Section, we utilize a set of $N$ distributed autonomous agents. Instead of being user-defined, the number of agents $N$ is determined by the image size and its content. The goal is to ensure, that the entire image is covered with agents, so that all information anywhere on the image is considered. As each agent locks a patch of size $5 \times 5$ around its final position (see Subsection 4.1), $N$ ranges between $\frac{H \times W}{5} \leq N \leq \frac{H \times W}{3}$, with $H$ and $W$ being the image height and width.

The algorithm begins with the movement phase, in which the agents iteratively re-position themselves until they have found the locally most meaningful position (see 4.2). Once an agent has reached its final position, it analyzes the surrounding pixels to gather local information (see 4.1.1). In the end of the movement phase, the final value of $N$ is obtained. In the next phase, the agents acquire information of their neighbored agents and build an initial estimation of the position and orientation of a contour within their local neighborhood (see 4.3). In the last phase, the agents interact to iteratively adjust their model to their neighbors' and thus grow from local models towards global contour estimates (see 4.4). Figure 2 shows an overview of the procedural flow of the algorithm.

For communication purposes, each agent is assigned a unique $I D$, from $1 \ldots N$.

\subsection{Local View}

For information acquisition, positioning, and contour estimation, each agent features three different windows, an analyzing window $W_{A}$ of size $3 \times 3$, a positioning window $W_{P}$ of size $5 \times 5$, and a communication window $W_{C}$ of size $11 \times 11$, each centered around the agent's position. Figure 3 illustrates the extent of each of the three windows, centered around an agent. The choice of the window sizes allows a special interplay between the agents, which will be explained in detail in the following Subsections. 


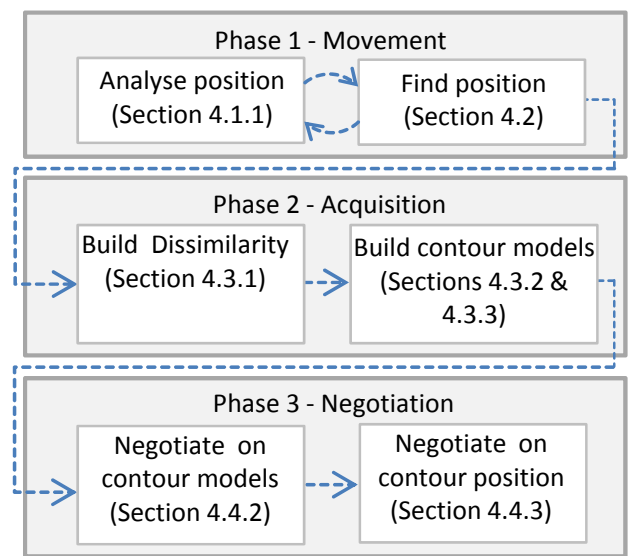

Figure 2: Overview of the three phases of the agents. The single steps are explained in the corresponding Subsections.

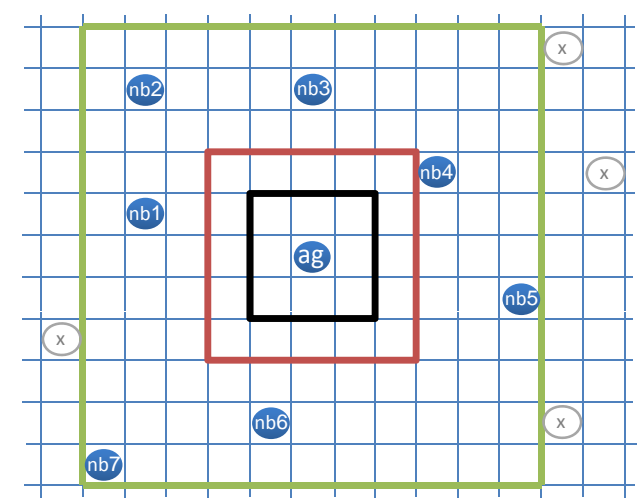

Figure 3: Local view of an agent ag with its three windows, the analyzing window $W_{A}$ (black), the positioning window $W_{P}$ (red), and the communication window $W_{C}$ (green). Within $W_{C}$ the agent can interact with its neighbors $n b 1$ to $n b 7$. Agents outside $W_{C}$ are displayed with an $x$ and cannot be contacted by ag.

\subsubsection{Analyzing Window}

Every agent ag can directly access image data inside its analyzing window $W_{A}$, which is centered at position $\left(x c_{a g}, y c_{a g}\right)$. The size of $W_{A}$ is considerably small to allow accurate localization and discovery of arbitrary and capillary contour shapes.

The information each agents is gathering is firstly the arithmetic mean $M_{W_{A}}^{c h}$ of the nine pixels inside $W_{A}$ via

$$
M_{W_{A}}^{c h}(a g)=\frac{1}{9} \sum_{i=-1}^{1} \sum_{j=-1}^{1} I_{c h}\left(x c_{a g}+i, y c_{a g}+j\right),
$$

for each color channel $c h$, with $I_{c h}(x, y)$ being the image values of channel ch at the pixel position $x, y$. Secondly, each agent calculates the mean edge strength $E_{W_{A}}$ using

$$
E_{W_{A}}(a g)=\frac{1}{9} \sum_{i=-1}^{1} \sum_{j=-1}^{1} G\left(x c_{a g}+i, y c_{a g}+j\right),
$$

with $G(x, y)$ being the magnitude of the image gradient at the pixel position $x, y$, computed by

$$
G(x, y)=\sqrt{I_{x}(x, y)^{2}+I_{y}(x, y)^{2}} .
$$


The values of $I_{x}$ and $I_{y}$ are the discretized partial derivative images in $x$ and $y$ direction, which are computed on the grayscale image via some standard approach like Sobel, or Prewitt.

\subsubsection{Positioning Window}

The positioning window $W_{P}$ describes the agents' movement capability, which will be explained in Subsection 4.2. Once an agent has discovered its final position, it blocks its area in the size of $W_{P}$, prohibiting any neighbored agent to move closer than 3 pixels towards the agent. The neighbored agent therefore is forced to search its final position outside the blocked area. Thus the size of $W_{P}$ ensures non-overlapping $W_{A}$ windows. Figure 4 shows the possible distances between an agent ag and its neighbor $n b x$ in $x$-direction. It is plain to see that, if neighbor $n b x$ settles on a position with a distance between 3 and 5 pixels, it will stay a direct neighbor of ag (examples $a$ to $c$ in Figure 4). A distance $\geq 6$ pixels in one direction will cause another neighbor nby to move in the gap between the positioning windows of $a g$ and $n b x$ (example $d$ ). This positioning schema forces agents to settle along the contours everywhere in the image, thus making contours describable by the agents.

\subsubsection{Communication Window}

Gathering image information within small windows like $W_{A}$ is generally prone to the typical errors in contour or boundary detection described in Section 1. We tackle this shortcoming via the communication window, that enables the agents to interpret the small local information in a broader context to eventually robustify the agents' collective decision. Furthermore, the communication principle allows to establish global contour estimations from the local information. The size of $W_{C}$ descends from the size of $W_{P}$, as its size ensures that there is at least one neighbor, i.e. a communication partner in each main direction surrounding an agent. As shown in Subsection 4.1.2 and Figure 4, the distance between an agent and a neighbor in any direction is 5 pixels at most, which coevally marks the needed extent of $W_{C}$ in one direction.

The communication window $W_{C}$ limits the area, in which an agent can find and contact neighbored agents. Each agent can communicate with all agents inside its communication window to access their model information. Any agent also collects the neighbored agents' IDs and their positions to relate them geometrically to its own position. The geometric information is utilized when the contour models are built. The access of neighbored agents constitutes a communication that resembles a spatially limited blackboard communication. To affirm their final connections, the agents furthermore use a direct communication, checking if their connection requests, i.e. their contour estimates, are acknowledged by the desired neighbor. Only connections that are confirmed by all connected agents are taken into account in the final contour image.

a
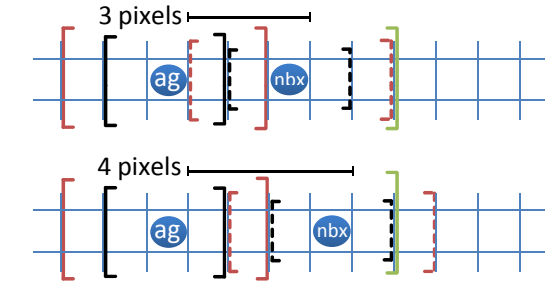

C
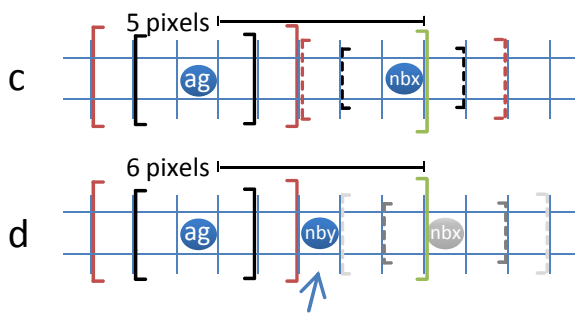

Figure 4: Possible pixel distances of neighbors in $x$ direction, minimum 3 and maximum 5 . The black, red, and green solid lines refer to $W_{A}, W_{P}$, and $W_{C}$ of the agent. The black and red dashed lines show the $W_{A}$ and $W_{P}$ of a neighbor $n b x$. 


\subsection{Movement}

Initially the agents are distributed equally spaced over the entire image. The movement follows the goal of positioning the agents on image locations, which are possibly meaningful for contour detection. As a hint towards the final contours, the mean edge strength information $E_{W_{A}}$ of Equation (2) is utilized. Every agent moves over the image iteratively checking the $E_{W_{A}}$ values inside its $W_{A}$, centered at each pixel position $\left(x c_{a g}, y c_{a g}\right)$ within its $W_{P}$. Subsequently each agent moves to the maximum inside $W_{P}$ and re-centers the $W_{P}$, until it discovers a local maximum edge strength. Let $t$ denote the current iteration, the movement rule can be expressed by

$$
\left(x c_{a g}, y c_{a g}\right)^{(t)}=\left(x c_{a g}+i^{*}, y c_{a g}+j^{*}\right)^{(t-1)},
$$

with $i^{*}, j^{*}$ computed by

$$
\left(i^{*}, j^{*}\right)=\underset{i, j \in \mathbb{Z}^{[-2,2]}}{\arg \max } E_{W_{A}}\left(x c_{a g}+i, y c_{a g}+j\right)
$$

A local maximum is detected, if the position does not change, i.e.

$$
\left(x c_{a g}, y c_{a g}\right)^{(t)}=\left(x c_{a g}, y c_{a g}\right)^{(t-1)}
$$

is fulfilled. When an agent reaches the final position, it locks the area of the size of $W_{P}$ around its position, so any other agent cannot get closer towards the locked position.

When the entire image is covered by agents, each holding a local maximum position regarding the edge strength, the movement phase stops.

\subsection{Initial Contour Model}

Each finally positioned agent tries to build an initial contour model. It therefore estimates the course and the position of a possible contour or boundary within its communication window. Furthermore, it evaluates the strength or probability of the existence of the assessed contour. For this purpose, the agent calculates three major fitness values, i.e. the agent dissimilarity value $D$, contour model fitness $M F$, and the contour position fitness PF.

\subsubsection{Agent Dissimilarity Value}

Each agent calculates the agent dissimilarity value $D_{a g}\left(n b_{i}\right)$ to each neighbored agent $n b_{i}, i=1, \ldots, n$, with $n$ being the number of neighbored agents within $W_{C}$. This measure describes the agent's dissimilarity to the neighbor utilizing the information gathered in each agent's $W_{A}$. A high $D_{a g}\left(n b_{i}\right)$ value means a high dissimilarity.

The agent dissimilarity value is calculated via

$$
\begin{aligned}
D_{a g}\left(n b_{i}\right) & =\alpha \cdot \frac{1}{C H} \cdot \sum_{c h}^{c H} \frac{\left(M_{W_{A}}^{c h}\left(n b_{i}\right)-M_{W_{A}}^{c h}(a g)\right)^{2}}{M_{W_{A}}^{c h}\left(n b_{i}\right)+M_{W_{A}}^{c h}(a g)} \\
& +\beta \cdot \frac{\left(E_{W_{A}}\left(n b_{i}\right)-E_{W_{A}}(a g)\right)^{2}}{E_{W_{A}}\left(n b_{i}\right)-E_{W_{A}}(a g)},
\end{aligned}
$$

with $M_{W_{A}}(j)$ and $E_{W_{A}}(j)$ being the mean image value and the mean edge strength value of the $W_{A}$ of agent $j$, as defined in Subsection 4.1.1, ch being the the current color channel, running from $1 \ldots \mathrm{CH}$, and $\mathrm{CH}$ being the number of image channels. In $R G B$ color images $\mathrm{CH}=3$ and in grayscale images $\mathrm{CH}=1$. The factors $\alpha$ and $\beta$ allow weighting of color and edge information.

The dissimilarity values $D_{a g}\left(n b_{i}\right)$ enable the agent to compare its image information to that of each neighbor. In Equation (7) we adapt a formula, which is widely used as a dissimilarity measure for histograms (see e.g. [34]). This method emphasizes small image discontinuities in smooth regions, while it attenuates dissimilarities in textured regions. 


\subsubsection{Contour Model Fitness}

Using its neighbored agents, each agent establishes several contour models, which describe an estimation of a contour's possible orientation or course. This is exemplarily shown in Figure 5. To build a contour model $m$, the agent first connects itself to two neighbored agents. The connected agents, marked as $C_{a g}^{m}$, are supposed to have a considerably small agent dissimilarity value. Such a connection splits the agent's communication window into two regions $A_{a g}^{m}$ and $B_{a g}^{m}$, with an arbitrary number of neighbored agents positioned inside each of them. The agent allocates each neighbor to a region using the relative position and geometry information. In a second step, the $D_{a g}$ values of the neighbors in $A_{a g}^{m}$ and $B_{a g}^{m}$ are used to determine the region most separated to the agent and its two connected neighbors. Hence, high dissimilarity values in a region advert to a separation.

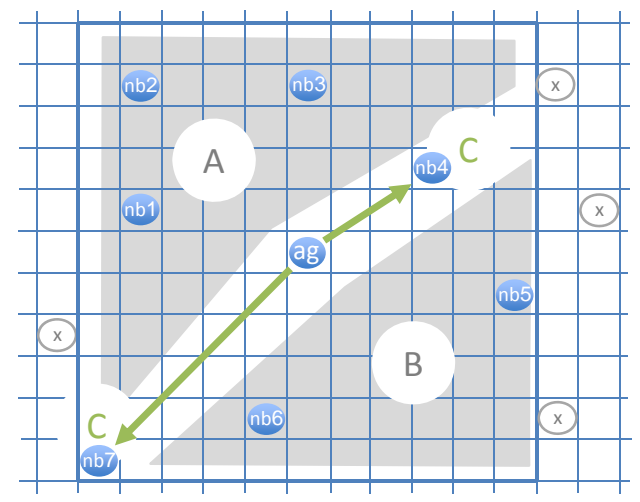

Figure 5: A contour model $m$ of agent ag, with all neighbors $n b 1 \ldots n b 7$ marked either as connected $C_{a g}^{m}$, or as belonging to region $A_{a g}^{m}$, or respectively region $B_{a g}^{m}$.

Consequentially, a contour model fitness $M F$ is calculated by

$$
M F_{a g}^{m}=\max \left(M D_{B_{a g}^{m}}, M D_{A_{a g}^{m}}\right)-M D_{C_{a g}^{m}},
$$

with $m$ being current model running from $m=1, \ldots, M . M D_{B_{a g}^{m}}, M D_{A_{a g}^{m}}, M D_{C_{a g}^{m}}$ are the mean of the $D_{a g}$ values of the neighbors, positioned either in $A_{a g}^{m}, B_{a g}^{m}$ or marked as connection $C_{a g}^{m}$. The maximum number of possible models $M$ for each agent is a user-defined number that can be used to limit the computational amount. Only the most promising models, estimated via the $D_{a g}$ value of the connected neighbors are taken into account. From the set of $M$ models, all agents pick the model with the maximum $M F$ as a starting point.

Eventually, each model $m$ is described with

- the set $C_{a g}^{m}$, which consists of the IDs of the two connected neighbors,

- the sets $A_{a g}^{m}$ and $B_{a g}^{m}$, holding the agents' IDs that lie in the two regions separated by $C_{a g}^{m}$,

- the information, which region is the most separated one, and

- the fitness $M F_{a g}^{m}$ evaluating the quality of each model.

Finally, we want to mention that $C_{a g}^{m}, A_{a g}^{m}$ and $B_{a g}^{m}$ are non-intersecting sets, i.e.

$$
C_{a g}^{m} \cap A_{a g}^{m}=C_{a g}^{m} \cap B_{a g}^{m}=A_{a g}^{m} \cap B_{a g}^{m}=\emptyset
$$

\subsubsection{Contour Position Fitness}

As stated above, the contour model predominantly describes how a contour or boundary is orientated within an agent's neighborhood, and which region is separated from the agent. However, each agent additionally has to estimate the contour's exact location within the neighborhood, i.e. whether the assessed contour goes through the agent and its two connections, or possibly parallel through the neighbored agents in the separated region. As the dissimilarity values $D_{a g}$ and the mean edge strength $E_{W_{A}}$ are highest in each direction right upon 
a contour, each agent calculates a contour position fitness $P F$, simply by

$$
P F_{a g}=\frac{1}{n} \cdot \sum_{i=1}^{n} D_{a g}\left(n b_{i}\right)+E_{W_{A}}(a g),
$$

with $D_{a g}\left(n b_{i}\right)$ being the agent dissimilarity values of each neighbor $n b_{i}, i=1, \ldots, n$, and $n$ being the number of neighbored agents.

\subsection{From Local Models to Global Contours}

The use of the neighbored agents' information natively causes a dependance between the initial contour models of each agent. However, in certain situations the initial model picked by an agent can be erroneous due to misleading local image information. Experiments showed that in those cases the models picked by the agents only have slightly higher MF values than the models that are globally desired, or respectively regarded as correct. Furthermore, there is a subset of surrounding agents, which initially prefer the correct course of the contour. Thus, in a further step, the agents align and adjust their individual models to those of their neighbors. This exhibits the second aspect of communication besides each agent's access to its neighbors.

To accomplish the alignment of the contour estimations, the agents use communication, i.e. they share and compare their estimates with each other and negotiate about which model to follow. Thus, the final aligned models are the best compromise within the neighborhood.

When aligned, the contour model yields agents located on contours to connect with neighbors along this contour. Additionally, it forces agents near this contour to construct models with connections parallel to that contour. Therefore, a contour is estimated not only by the agents placed on it, but also by the agents placed parallel to it in its surrounding. This is a key point of this algorithm, increasing robustness in ambiguous image regions.

\subsubsection{Conflicts between Contour Models}

While the agents' contour models are unaligned, two main conflicts arise between the agents, which are illustrated in Figure 6.

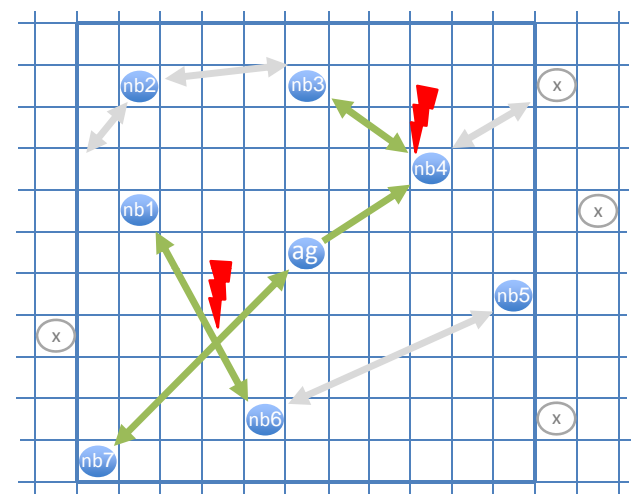

Figure 6: Example of the possible conflicts of the agents' models. The arrows mark the connection requests of the agents. Multiple connection requests between $a g, n b 4$, and $n b 3$, and crossing contours between the connections $a g \leftrightarrow n b 7$ and $n b 1 \leftrightarrow n b 6$.

The first conflict is referred to as multiple connection requests. It originates, when two or more agents want to connect to the same neighbor, which is shown in Figure 6 . Here, both ag and $n b 3$ request $n b 4$ for a connection, but $n b 4$ initially connects to $n b 3$. This can be stated as

$$
n b i \in C_{a g}^{m_{k}} \wedge a g \notin C_{n b i}^{m_{l}} \text {, }
$$


with $C$ being the connection sets described in Subsection 4.3.2, and $k$ and $/$ being the currently chosen models of ag and $n b i$, respectively. In this example $n b i$ is $n b 4$.

The second conflict which appears is called crossing contours, and describes the situation, where two connections, and thus two contour estimates cross each other. As both connection estimations also imply that there are two regions which are not connected to each other, crossing connections must display a conflict. This is illustrated in Figure 6 with the connection of $a g$ and $n b 7$ crossing the connection of $n b 1$ and $n b 6$. For a neighbor $n b i$ this can be stated as

$$
n b i \in q_{a g}^{m_{k}} \wedge C_{n b i}^{m_{l}} \in r_{a g}^{m_{k}}
$$

with $q, r \in[A, B], q \neq r$, and $k$ and $/$ being the currently chosen models of ag and $n b i$, respectively. In this example $n b i$ is $n b 6$ and $n b 1$.

\subsubsection{Negotiation on Contour Models}

In order to solve the conflicts described in Subsection 4.4.1, each agent involved searches in its set of possible models $m=1, \ldots, M$ for the best model that is not conflicting. Thus, in case of a multiple connection request between ag and its neighbor $n b i$, the agent ag takes the model $m_{o}^{*}$, which holds

$$
m_{o}^{*}=\underset{m_{o} \mid n b i \notin C_{a g}^{m_{o}}}{\arg \max }\left(M F_{a g}^{m_{o}}\right) .
$$

Analogously, nbi chooses the model $m_{p}^{*}$, which holds

$$
m_{p}^{*}=\underset{m_{p} \mid a g \in C_{n b i}^{m_{p}}}{\arg \max }\left(M F_{n b i}^{m_{p}}\right)
$$

In Figure $6, n b i$ is $n b 4$. The crossing contours conflict follows the same mechanism, which means e.g. for ag and $n b 6$ in Figure 6 that both search for the best model $m_{o}^{*}$ and $m_{p}^{*}$, which do not meet the condition stated in Equation (12).

The agents corporately decide, which agent has to change its model, by comparing the model fitness of the old models $m_{k}$ and $m_{l}$ to those of the new models $m_{o}^{*}$ and $m_{p}^{*}$. Therefore, each involved agent calculates the model fitness difference $\delta_{a g}=M F_{a g}^{m_{k}}-M F_{a g}^{m_{o}^{*}}$ and $\delta_{n b i}=M F_{n b i}^{m_{l}}-M F_{n b i}^{m_{p}^{*}}$. The agent with the smaller difference has to change from the old to the new model, so if $\delta_{n b i}<\delta_{a g}$, then $m_{l} \rightarrow m_{p}^{*}$, and $m_{k} \rightarrow m_{o}^{*}$ otherwise.

This procedure ensures that the overall fitness within the neighborhood is maximized. If an agent cannot find a model to solve the conflict, the value $M F_{a g}^{m_{o}^{*}}$, or respectively $M F_{n b i}^{m_{p}^{*}}$ is set to zero, causing the maximum possible difference. In this way, agents change their models until no conflicts occur any more. Agents with no acknowledged connections, or no possible model left to choose will be regarded as deactivated and no longer be considered for contour detection in the subsequent stage. This desired effect takes place within regions where agents either cannot agree to a final contour model, as there is none, or their final contour strength is negligibly small compared to the correct contours or boundaries. Hence, the algorithm is self-regulating to a certain degree.

\subsubsection{Negotiation on Contour Position}

When all conflicts are solved, the agents will either have established aligned contour models, which run parallel along region borders, or be deactivated. In order to decide for the exact position, the remaining active agents sum the PF values of themselves and their connections and compare it to the parallel model that lies in the direction of their most separated region. Each agent adds its final $M F_{a g}^{m}$ to the agent with the maximum $P F$, which can also be the agent itself, and its two connections. Thus, parallel models support each other and strengthen the final position.

The final contour image corresponds to the summed position fitness of each agent and its connected neighbors. As stated above, only agents with connections that are without conflict and acknowledged by the respective connection partners are taken into account. 


\section{Experiments and Results}

In this Section we show some results of our algorithm achieved in task of boundary detection. We will first comment on evaluation metrics and state our decision on the metrics that we applied. Then we will investigate and discuss the results of our algorithm in detail utilizing example images. Furthermore, we will deliver a comparison to methods from the literature which used the same evaluation metrics, and in the end, we will discuss the computational efficiency of our method.

\subsection{Evaluation Dataset and Metrics}

As described in the introduction, a major problem when it comes to evaluate boundary detection or image segmentation algorithms, is the need of a ground truth, and coevally the lack of uniqueness of such. One of the most extensive and widely used image collections for this purpose is the Berkeley Segmentation Dataset (BSDS) [1], which provides a set of 500 natural images of size $481 \times 321$, each along with 5-10 humangenerated segmentations, which serve as ground-truth.

General evaluation metrics for image segmentation are for example the Probabilistic Rand Index [35], the Jaccard index, or the Object-level Consistency Error OCE [36]. Such metrics are usually based on the amount of overlap between ground-truth segments and segments detected by the algorithm. They differ for example in the used distance measures, the punishment for under- or oversegmentation, or the way the overlap is weighted with regard to the segment sizes. However, the metrics mentioned above demand image regions with closed boundaries that are fully segmented from each other, so any pixel in the image can be assigned to one segment exclusively. Therefore, those metrics cannot be applied to the topic of boundary detection, as such algorithms produce potentially non-closed contour estimates rather than segmented regions. Nevertheless, boundary based evaluation metrics exist, like Pratt's Figure of Merit (FoM) [37], Precision-Recall Curves (PRC) [1], and Receiver-Operator-Characteristics (ROC) [38], whereas the PRC-based benchmark system in [1] allows for comparison of region-based segmentation and contour detection methods in the same framework. Those methods usually measure the coincidence and the accuracy of boundary or edge pixels compared to groundtruth boundaries.

Pratt's Figure of Merit is a distance transform between the ground-truth and the evaluated boundary map binarized at varying thresholds. It measures the detection, localization, and spurious response, which means that the score is influenced by all edges being found, all edges being placed in the correct location, and false alarms. The FoM is computed by

$$
\text { FoM }=\frac{1}{\max \left(N_{G T}, N_{\text {res }}\right)} \sum_{k=1}^{N_{\text {res }}} \frac{1}{1+a d(k)^{2}},
$$

where $N_{G T}$ is the number of ground-truth edge pixels, $N_{\text {res }}$ is the number of edges pixels detected by the algorithm, $d(k)$ denotes the distance from the $k$ th detected edge pixel to the nearest ground-truth edge pixel, and $a$ is a scaling constant, set to $1 / 9$ as in Pratt's work. The metric ranges from 0 to 1 , with 1 being the best obtainable result, which means that the ground-truth and the detected result are identical. As the threshold is varied, the best result obtained is used for evaluation [37].

Along with the BSDS image database, [1] delivers a benchmark, in which the ground-truth data is compared to machine generated output using Precision-Recall Curves. Precision measures the probability that a machinegenerated boundary pixel is a true boundary pixel, while Recall gauges the probability that a true boundary pixel is detected, i.e. the amount of ground-truth captured by an algorithm. Again, in order to obtain binarized edge maps from the algorithm's edge fitness output, Precision and Recall are calculated for a set of varied thresholds, which captures the trade-off between the accuracy and noise, i.e. the false-positives and the true-negatives.

To approximate the Precision-Recall Curves' optimal trade-off, the F-Measure is calculated for each threshold. The F-measure is the harmonic mean of Precision and Recall, calculated by

$$
F=\frac{2 \cdot \text { Precision } \cdot \text { Recall }}{\text { Precision }+ \text { Recall }}
$$

Another well-known metric in image evaluation is the Receiver-Operator-Characteristic, or ROC-Curves, whose axes are called Fallout and Recall. These metrics are comparable to the Precision-Recall metric. The 
Recall is defined identically in both metrics. Fallout measures the probability that a true negative was labeled a false positive. Just as the PR-Curve, the ROC-Curve is a graphical plot that displays the performance of a binary classifier system with a varied binarization threshold. However, the definition of the Fallout makes the ROC-Curves dependent on the image resolution, while the Precision-Recall Curves have the advantage of being scale-invariant [39] and are therefore preferred.

The BSDS benchmark delivers three major quantities: first the Optimal Dataset Scale (ODS), which is best F-measure on the dataset for a fixed scale or threshold, second the Optimal Image Scale (OIS), which is the aggregate F-measure on the dataset for the best scale in each image, and third the Average Precision (AP) on the full Recall range, which is equivalently the area under the Precision-Recall Curve.

\subsection{Examples and Discussion}

The BSDS image dataset is partitioned in images for training, testing, and validation. For the evaluation, we ran the algorithm on the test- and validation-set of 300 images overall. We used the training set to empirically find the optimal parameters for the weighting factors $\alpha$ and $\beta$ of the agent dissimilarity values, which were set to $\alpha=0.35$ and $\beta=0.65$.

Initial experiments revealed that the negotiation mechanism delivers an overall improvement of 0.01 for the ODS and OIS values compared to the initial connection estimates. This indicates that the initial Boundary Model estimates, relying on the Dissimilarity Value (see Subsection 4.3.1) and on the rule that only connections acknowledged by the neighbors are used, are generally sound. While the negotiation does not significantly improve the overall result in the complete dataset, we could observe slight improvements in ambiguous image areas, where the initial estimates of the agents were misleaded by e.g. textures.

Figures 7 to 10 present example images categorized into four different categories, each along with the ground-truth, our resulting boundary images, and the corresponding Precision-Recall Curves with the optimal F-measure. Figure 7 shows example images, where the algorithm performs well, as the picture content is quite distinguishable and consists of salient regions. The performance on the images displayed in the introduction (see Figure 8) is similar to the average performance over the entire image set. The image content includes a balanced mixture of strong edges, salient regions, and also cluttered or textured regions.

The three example images of Figure 9 contain a considerable amount of textured regions, which leads to a significant performance loss of our algorithm. This is due to the fact, that textures are not explicitly considered when calculating the boundary estimates. An improvement is to expect, when the agents are extended to incorporate texture cues like Textons, or Local Binary Patterns into the Dissimilarity Value formula. Furthermore, examining the image information inside an agent's neighborhood for repeating patterns texture-like structures seems promising. We will investigate both approaches in future work.

In [39] the authors argue that contour detection, while being closely related, is not perfectly the same as boundary detection. They state that contours, which might be important in terms of the image content do not necessarily have to be region boundaries, and might thus be excluded in boundary detection. The three example images in Figure 10 illustrate the difference between boundaries and contours. It is obvious that our algorithm detects contours rather than boundaries, e.g. the single windows in the left picture, or the black and white stripes of the zebras. While extracting these salient contours might be desirable in some applications, it significantly degrades the performance of our algorithm with regard to boundary detection, as can be seen in the PR-Curves. To a certain extent, these misleading detections might supposedly be alleviated by considering textures, as stated above. For further elimination, additional information, e.g. in form of object or appearance models, knowledge, or training might become necessary.

\subsection{Comparison}

Table 1 shows the results for the FoM metric of our boundary detector, in comparison with the method from [3], and the Canny Detector. It displays the mean FoM and standard deviation, calculated over the BSDS test dataset. The method from [3] expectably performs best in terms of the FoM measure, our algorithm operates slightly better than the Canny edge detector.

The lower part of Table 2 confirms the trend of the FoM measure, with the method of [3] performing best, followed by our algorithm, which works better than the Canny method. Table 2 additionally shows a comparison 


\begin{tabular}{|r|cc|}
\hline Method & mean FoM & Std.Dev \\
\hline gPb-owt-ucm [3] & 0.6815 & \pm 0.11422 \\
our algorithm & 0.50242 & \pm 0.11611 \\
Canny & 0.49208 & \pm 0.13758 \\
\hline
\end{tabular}

Table 1: Results of Pratt's Figure of Merit (FoM) measure for the gPb-owt-ucm method described in [3], our algorithm and the Canny detector, mean and standard deviation calculated over the BSDS 500 test dataset.

of other segmentation methods, provided by [3]. The compared methods besides $g P b$ are originally described in [40], [5], [6], and [9].

\begin{tabular}{|c|c|c|c||c|c|c|}
\cline { 2 - 7 } \multicolumn{1}{c|}{} & \multicolumn{3}{c||}{ BSDS300 } & \multicolumn{3}{c|}{ BSDS500 } \\
\cline { 2 - 7 } \multicolumn{1}{c|}{} & ODS & OIS & AP & ODS & OIS & AP \\
\hline Human & 0.79 & 0.79 & - & 0.80 & 0.80 & - \\
\hline [3] gPb-owt-ucm & 0.71 & 0.74 & 0.73 & 0.73 & 0.76 & 0.73 \\
[9] Mean Shift & 0.63 & 0.66 & 0.54 & 0.64 & 0.68 & 0.56 \\
[6] NCuts & 0.62 & 0.66 & 0.43 & 0.64 & 0.68 & 0.45 \\
[3] Canny-owt-ucm & 0.58 & 0.63 & 0.58 & 0.60 & 0.64 & 0.58 \\
[5] Felz-Hutt & 0.58 & 0.62 & 0.53 & 0.61 & 0.64 & 0.56 \\
[40] SWA & 0.56 & 0.59 & 0.54 & - & - & - \\
\hline gPb & 0.70 & 0.72 & 0.66 & 0.71 & 0.74 & 0.65 \\
our algorithm & $\mathbf{0 . 6 0}$ & $\mathbf{0 . 6 3}$ & $\mathbf{0 . 5 2}$ & $\mathbf{0 . 6 3}$ & $\mathbf{0 . 6 5}$ & $\mathbf{0 . 5 7}$ \\
Canny & 0.58 & $\mathbf{0 . 6 2}$ & 0.58 & 0.60 & 0.63 & 0.58 \\
\hline
\end{tabular}

Table 2: Boundary benchmark results on the BSDS300 and BSDS500, for seven different segmentation methods in the upper Table and two contour detectors plus our algorithm in the lower Table, taken from [3]. As our method is a boundary detector, we added its results in the lower Table. The values represent the F-measures using an optimal scale for the entire dataset (ODS) or per image (OIS). The area-precision is expressed in (AP).

Our tests reveal results that are quite promising, keeping in mind, that e.g. the $\mathrm{gPb}$ method of [3] is a highly advanced and sophisticated concept, developed and refined over several years, which explicitly considers e.g. texture information and also benefits from training phases.

Similarly, most of the methods compared in Table 2 utilize further image cues. As stated in Section 5.2, our method does not yet explicitly apply texture information but we are confident, that we could further improve our results by integrating more image information.

\subsection{Computation time}

Two parameters have principal influence on the computation time of our algorithm. The first is the number of agents $N$, which in turn depends on the image size, resulting in $\frac{1}{3} \times H \times W$ as worst case. The second influence is the number of negotiations, where in a worst case scenario negotiations would run until any agent has checked all its possible models. The number of negotiations directly depends on the maximum number of possible models $M$. Thus, the runtime of the algorithm is linearly dependent as $O(N \cdot M)$.

We ran our algorithm on an Intel Core 2 Quad CPU Q6600, 2.39 Ghz and 3 GB of RAM, implemented in MATLAB. We used a mex-File to implement the negotiation part in $C$, besides that no further code optimization was utilized. Our algorithm ran with 5.74 seconds on average per $481 \times 321$-image. For comparison, the 
$g P b$-method from [3] demands around four minutes of of computation time for one image of the dataset using a regular $C$ implementation. As stated in [41], the computation time of the $g P b$-method was reduced to $1.8 \mathrm{sec}-$ onds utilizing parallelized GPU implementation. Thus, we believe that the computational speed of our method is advantageous when it comes to time-dependant applications.

\section{Conclusion}

In this work, we present a novel algorithm that uses a set of distributed agents and the principles of Swarm Intelligence to detect contours in images. Instead of optimizing a single existing task, our method seeks to analyze image content from scratch.

In a nutshell, the main contribution of our approach include (1) a moving rule, which by distributing the agents on the entire image in locally potentially meaningful positions creates a dynamic mesh; (2) a set of local analyze- and communication-tools that help agents to cooperatively decide on image contours based on their local environment; (3) a set of novel fitness functions, and an interactive negotiation rule leading to an overall best decision between the agents.

Contrary to recent methods, the algorithm does neither need any explicit object models nor training phases, or user-defined prior knowledge. The promising results of our experiments in the field of boundary detection indicate that the interaction of agents can lead from local segments to robust globally valid contours.

Further effort has to be spent on the agents' capability to deal with ambiguous regions, like texture and smooth transitions of regions. We will seek for methods to push the algorithm from contour to towards boundary detection. In order to step from boundary detection to image segmentation, we want to establish a version of the algorithm, in which agents can explicitly deal with contour junctions and also seek to close contours.

\section{References}

[1] D. Martin, C. Fowlkes, D. Tal, J. Malik, A database of human segmented natural images and its application to evaluating segmentation algorithms and measuring ecological statistics, in: International Conference on Computer Vision, 2001, pp. 416-423.

[2] D. Martin, C. Fowlkes, J. Malik, Learning to detect natural image boundaries using local brightness, color, and texture cues, IEEE Transactions on Pattern Analysis and Machine Intelligence 26 (5) (2004) 530 -549.

[3] P. Arbelaez, M. Maire, C. Fowlkes, J. Malik, Contour detection and hierarchical image segmentation, IEEE Transactions on Pattern Analysis and Machine Intelligence 33 (5) (2011) 898-916.

[4] S. Thilagamani, N. Shanthi, A survey on image segmentation through clustering, International Journal of Research and Reviews in Information Sciences 1 (1) (2011) 16-19.

[5] P. F. Felzenszwalb, D. P. Huttenlocher, Efficient graph-based image segmentation, International Journal of Computer Vision 59 (2) (2004) 167-181.

[6] T. Cour, F. Benezit, J. Shi, Spectral segmentation with multiscale graph decomposition, in: IEEE Conference on Computer Vision and Pattern Recognition, 2005, pp. 1124 - 1131.

[7] W. Cui, Z. Guan, Z. Zhang, An improved region growing algorithm for image segmentation, in: International Conference on Computer Science and Software Engineering, 2008, pp. 93 - 96.

[8] J. Chang, J. Fisher, Efficient mcmc sampling with implicit shape representations, in: IEEE Conference on Computer Vision and Pattern Recognition, 2011, pp. 2081 -2088.

[9] D. Comaniciu, P. Meer, Mean shift: a robust approach toward feature space analysis, IEEE Transactions on Pattern Analysis and Machine Intelligence 24 (5) (2002) 603 -619.

[10] J. Chen, T. Pappas, A. Mojsilovic, B. Rogowitz, Adaptive perceptual color-texture image segmentation, IEEE Transactions on Image Processing 14 (10) (2005) 1524 - 1536. 
[11] N. Senthilkumaran, R. Rajesh, Edge detection techniques for image segmentation - a survey of soft computing approaches, International Journal of Recent Trends in Engineering 1 (2) (2009) 250-254.

[12] Y. Ramadevi, T. Sridevi, B. Poornima, B. Kalyani, Segmentation and object recognition using edge detection techniques, International Journal of Computer Science \& Information Technology 2 (6) (2010) 153161.

[13] W. Ma, B. S. Manjunath, Edgeflow: a technique for boundary detection and image segmentation, IEEE Transactions on Image Processing 9 (8) (2000) 1375-1388.

[14] H. Wang, J. Oliensis, Generalizing edge detection to contour detection for image segmentation, Computer Vision and Image Understanding 114 (7) (2010) 731 - 744.

[15] K. Hammouche, M. Diaf, P. Siarry, A multilevel automatic thresholding method based on a genetic algorithm for a fast image segmentation, Comput. Vis. Image Underst. 109 (2) (2008) 163-175.

[16] S. Das, S. Sil, Kernel-induced fuzzy clustering of image pixels with an improved differential evolution algorithm, Inf. Sci. 180 (8) (2010) 1237-1256.

[17] C.-Y. Lee, J.-J. Leou, H.-H. Hsiao, Saliency-directed color image segmentation using modified particle swarm optimization, Signal Processing 92 (1) (2012) 1 - 18.

[18] L. Li, D. Li, Fuzzy entropy image segmentation based on particle swarm optimization, Progress in Natural Science 18 (9) (2008) 1167 - 1171.

[19] D. Feng, S. Wenkang, C. Liangzhou, D. Yong, Z. Zhenfu, Infrared image segmentation with 2-d maximum entropy method based on particle swarm optimization (pso), Pattern Recognition Letters 26 (5) (2005) 597-603.

[20] S. Saatchi, C.-c. Hung, Swarm Intelligence: Focus on Ant and Particle Swarm Optimization, Itech Education and Publishing, Vienna, Austria, 2007, Ch. Swarm intelligence-based image segmentation, pp. 163 178.

[21] S. A. Etemad, T. White, An ant-inspired algorithm for detection of image edge features, Applied Soft Computing 11 (8) (2011) $4883-4893$.

[22] M. Setayesh, M. Zhang, M. Johnston, Edge detection using constrained discrete particle swarm optimisation in noisy images, in: Evolutionary Computation (CEC), 2011 IEEE Congress on, 2011, pp. 246 -253.

[23] G. Beni, J. Wang, Swarm intelligence in cellular robotic systems, in: NATO Advanced Workshop on Robots and Biological Systems, 1989, pp. 26-30.

[24] W. Yong, C. Jun, Using ant swarm intelligence for data clustering analysis, in: 2nd IEEE International Conference on Computer Science and Information Technology, 2009, pp. 429 -432.

[25] A. V. Baterina, C. Oppus, Image edge detection using ant colony optimization, WSEAS Transactions on Signal Processing 6 (2) (2010) 58-67.

[26] A. Amali Asha, S. Victor, A. Lourdusamy, Feature extraction in medical image using ant colony optimization : A study, International Journal on Computer Science and Engineering 3 (2) (2011) 714-721.

[27] E. Lakehal, A swarm intelligence based approach for image feature extraction, in: International Conference on Multimedia Computing and Systems, 2009, pp. $31-35$.

[28] U. Kirchmaier, S. Hawe, K. Diepold, Dynamical information fusion of heterogeneous sensors for $3 d$ tracking using particle swarm optimization, Information Fusion 12 (4) (2011) 275 - 283.

[29] J. Kennedy, R. Eberhart, Particle swarm optimization, in: IEEE International Conference on Neural Networks, 1995, pp. 1942-1948. 
[30] A. Colorni, M. Dorigo, V. Maniezzo, Distributed optimization by ant colonies, in: Proceedings of the First European Conference on Artificial Life, 1992, pp. 123-142.

[31] F. M. A. Mohsen, A new optimization-based image segmentation method by particle swarm optimization, International Journal of Advanced Computer Science and Applications Special Isssue (Image Processing and Analysis) (2011) 10-18.

[32] C. White, G. Tagliarini, S. Narayan, An algorithm for swarm-based color image segmentation, in: IEEE SoutheastCon, 2004, pp. $84-89$.

[33] J. Liu, Y. Y. Tang, Adaptive image segmentation with distributed behavior-based agents, IEEE Transactions on Pattern Analysis and Machine Intelligence 21 (6) (1999) 544-551.

[34] X. Liu, D. Wang, A spectral histogram model for textons and texture discrimination, in: International Joint Conference on Neural Networks, Vol. 2, 2001, pp. $1083-1088$ vol.2.

[35] R. Unnikrishnan, C. Pantofaru, M. Hebert, Toward objective evaluation of image segmentation algorithms, IEEE Transactions on Pattern Analysis and Machine Intelligence 29 (6) (2007) 929-944.

[36] M. Polak, H. Zhang, M. Pi, An evaluation metric for image segmentation of multiple objects, Image and Vision Computing 27 (8) (2009) 1223-1227.

[37] W. K. Pratt, Digital Image Processing, 3rd Edition, John Wiley \& Sons Interscience, New York, 2001.

[38] K. Bowyer, C. Kranenburg, S. Dougherty, Edge detector evaluation using empirical roc curves, Computer Vision and Image Understanding 84 (1) (2001) 77 - 103.

[39] G. Papari, N. Petkov, Edge and line oriented contour detection: State of the art, Image and Vision Computing 29 (2-3) (2011) 79-103.

[40] E. Sharon, M. Galun, D. Sharon, R. Basri, A. Brandt, Hierarchy and adaptivity in segmenting visual scenes, Nature 42 (7104) (2006) 810-813.

[41] B. Catanzaro, B.-Y. Su, N. Sundaram, Y. Lee, M. Murphy, K. Keutzer, Efficient, high-quality image contour detection, in: Computer Vision, 2009 IEEE 12th International Conference on, 2009, pp. 2381 -2388. 

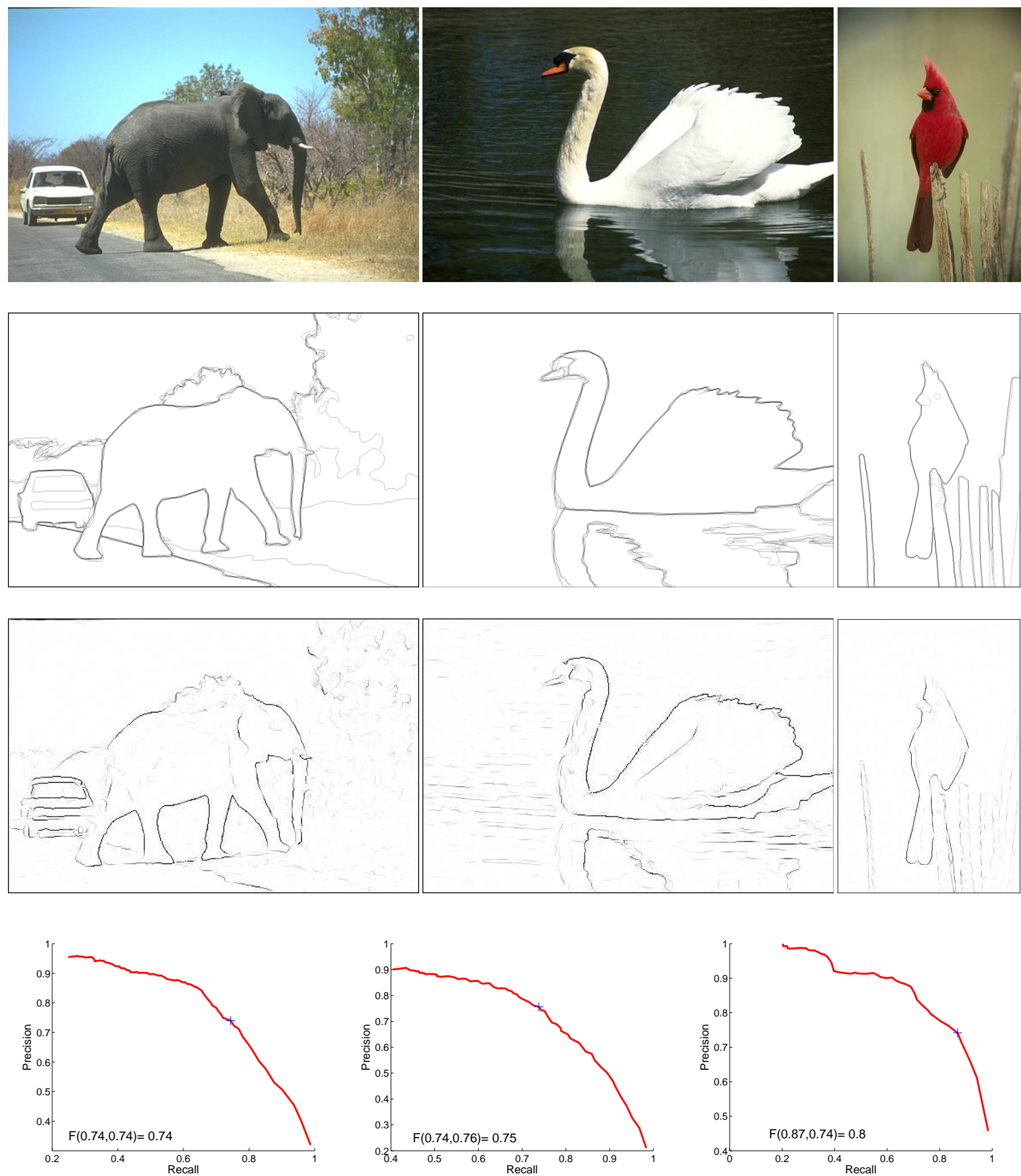

Figure 7: Three sample images with distinctive content from the Berkeley Image Segmentation Dataset (BSDS 500) [3]. From top to bottom: the original images, the summed ground-truth images, the result images and the precision-recall curves of our algorithm. 

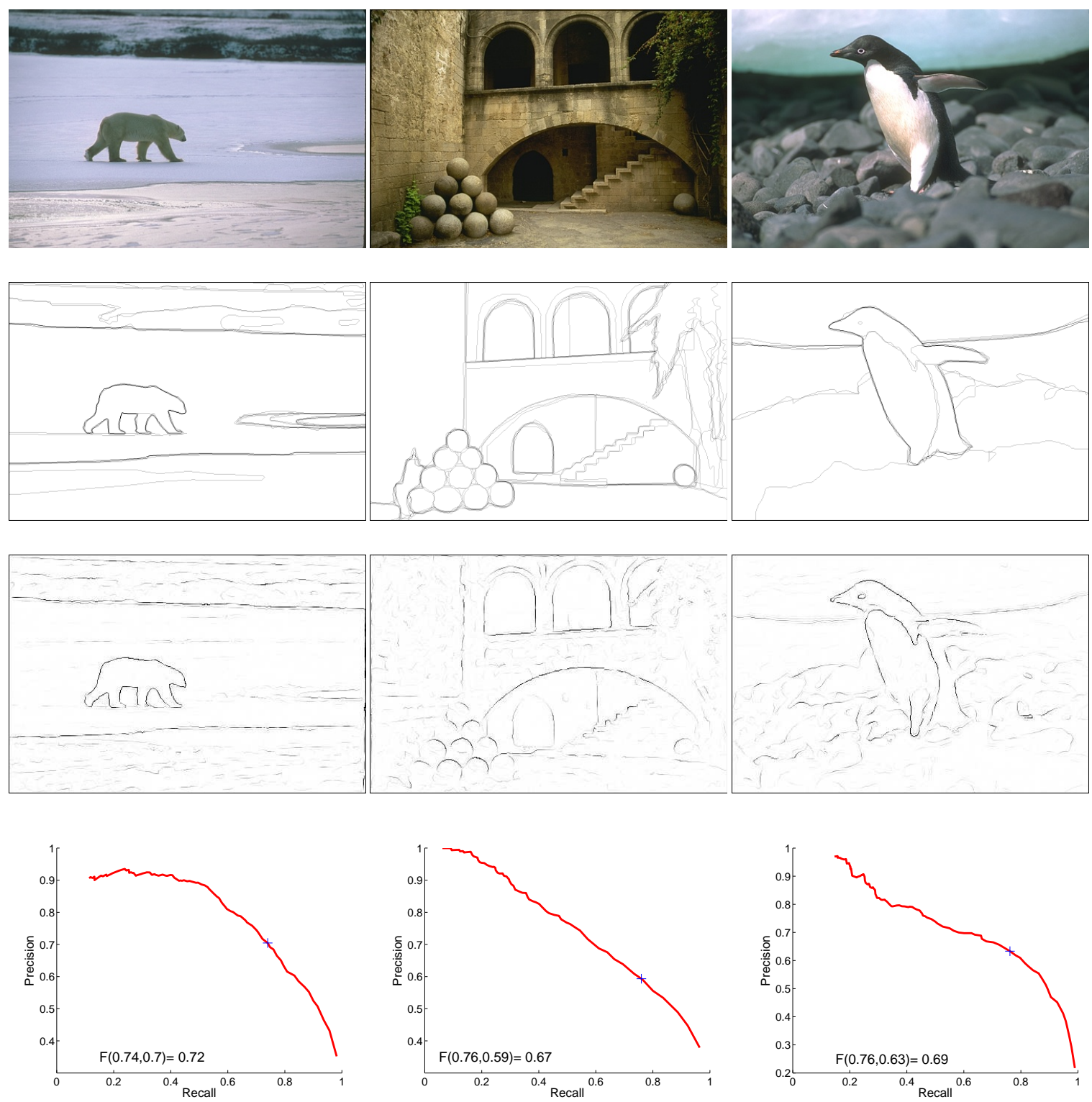

Figure 8: Three sample images from the introduction Section, which yield to average performance with regard to the overall results. Taken from the Berkeley Image Segmentation Dataset (BSDS 500) [3]. From top to bottom: the original images, the summed ground-truth images, the result images and the precision-recall curves of our algorithm. 

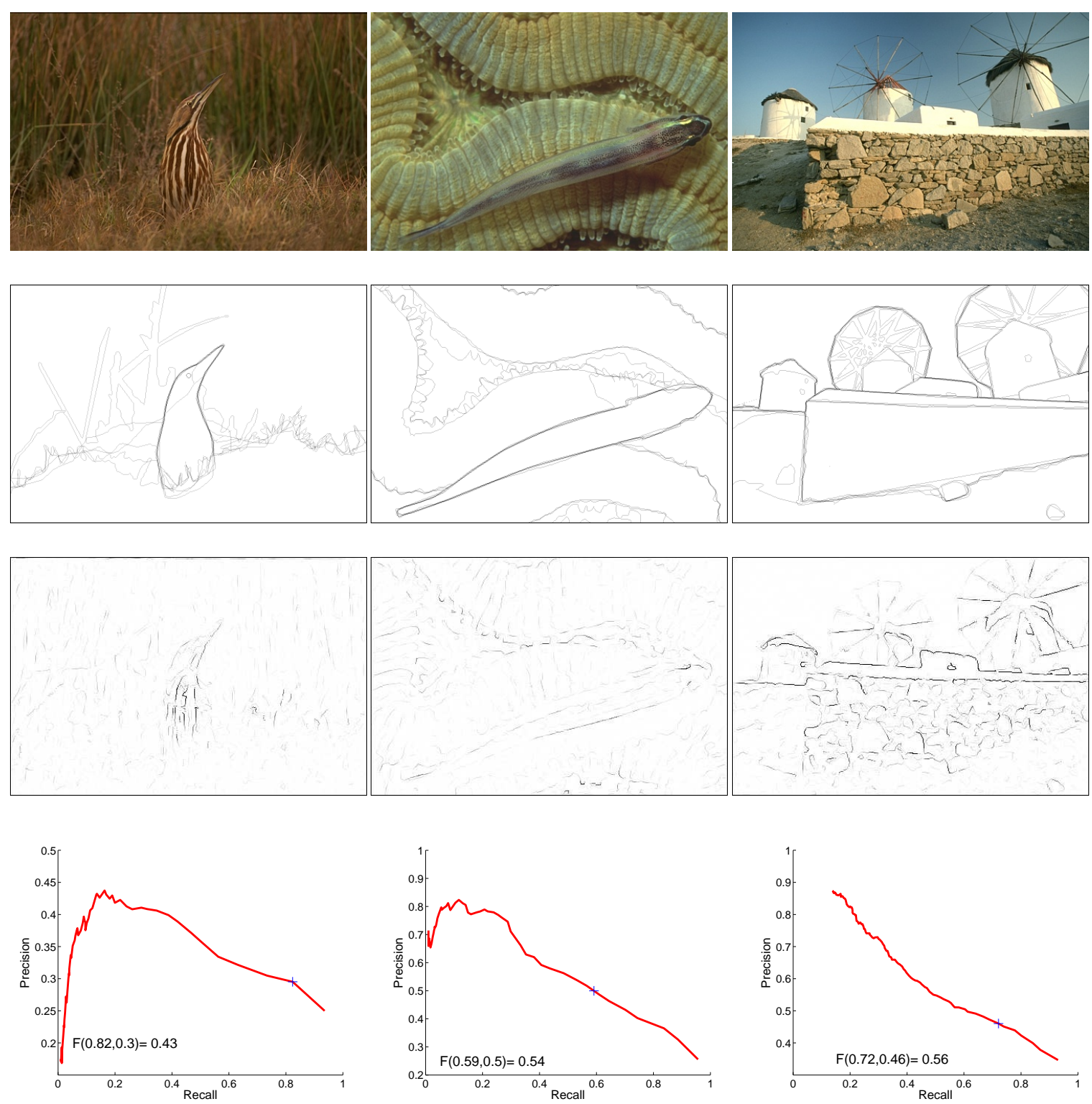

Figure 9: Three sample images containing strong texture. Taken from the Berkeley Image Segmentation Dataset (BSDS 500) [3]. From top to bottom: the original images, the summed ground-truth images, the result images and the precisionrecall curves of our algorithm. 

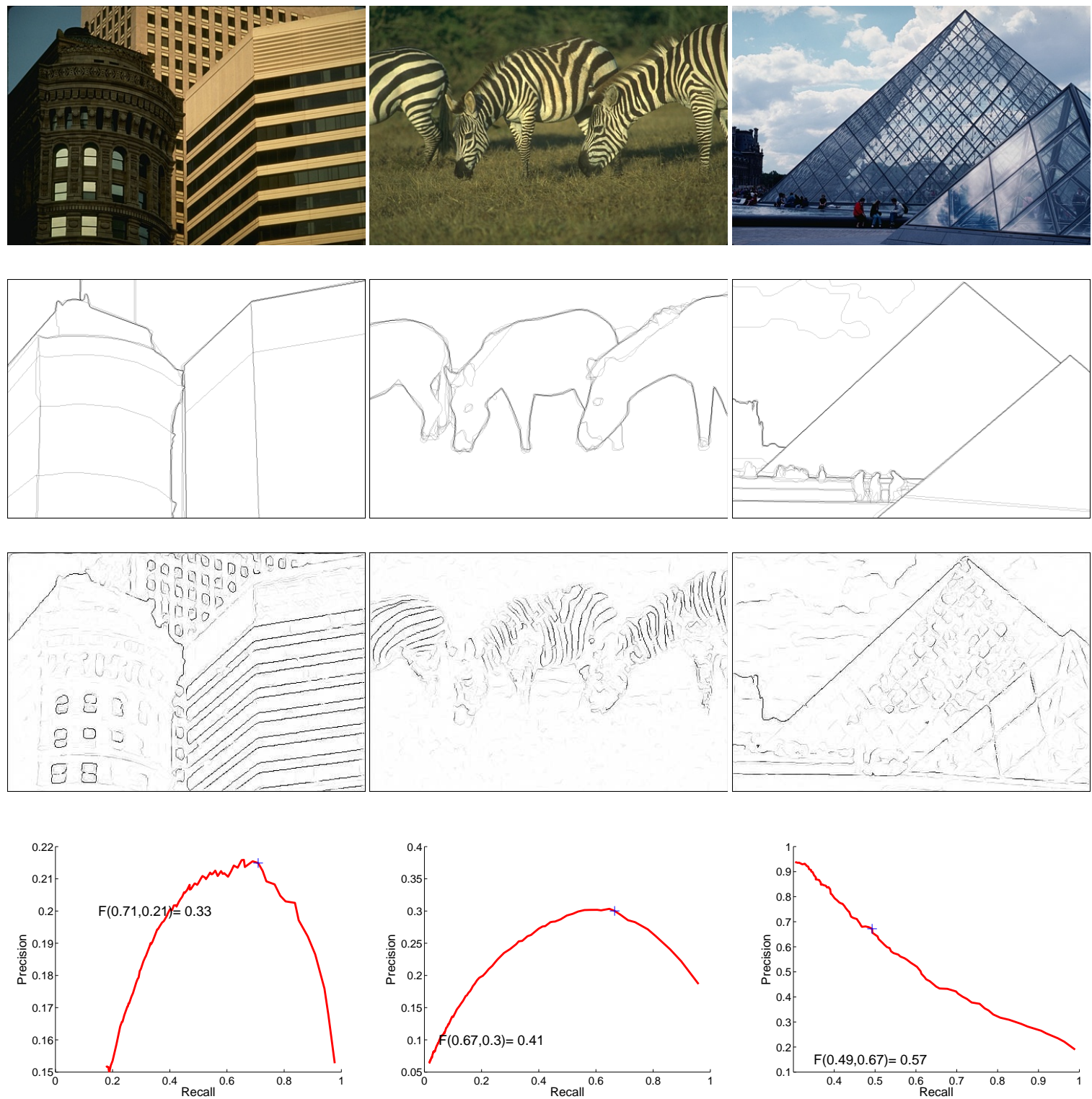

Figure 10: Three sample images containing strong contours that are not boundaries. Taken from the Berkeley Image Segmentation Dataset (BSDS 500) [3]. From top to bottom: the original images, the summed ground-truth images, the result images and the precision-recall curves of our algorithm. 